\title{
Acute pancreatitis in pregnancy - a case report and literature review
}

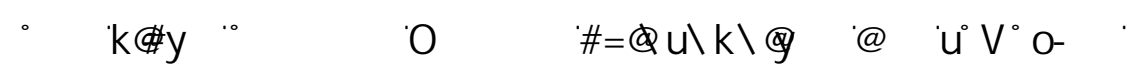

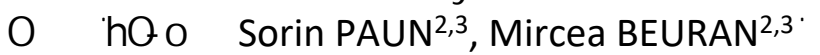

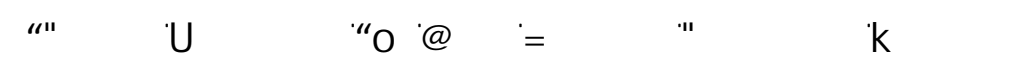

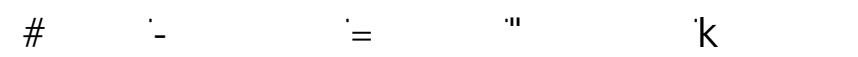

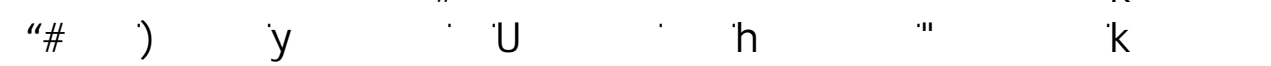

\begin{abstract}
Introduction. Acute pancreatitis in pregnancy is a rare condition with an approximate incidence of 1 over 1,000-12,000 pregnant women, but it is associated with increased incidence of maternal and fetal mortality. Can occur during any trimester but over half (52\%) occur during the third trimester and rarely during post-partum period. Difficulty in diagnosis and treatment put significantly in danger the maternal and fetal health. The treatment of pancreatitis in pregnancy should be conservative as far as possible with delaying the definitive treatment until after delivery.

Case report. We report a case of a 20-year-old woman, at 32 week of gestation who presented with severe pain confined to the upper abdomen and radiating to the back to the upper abdomen. Investigation undertaken led to the diagnosis of acute pancreatitis which was managed conservatively. She recovered within several days and then delivered a healthy baby.

Conclusions. Acute pancreatitis is rare in pregnancy, occurring most commonly in the third trimester. Pancreatitis in pregnancy remains a challenging clinical problem to manage, but if treated early, preterm labor can be avoided.
\end{abstract}

Keywords: acute pancreatitis, pregnancy, gallstones, prognosis, treatment

\section{INTRODUCTION}

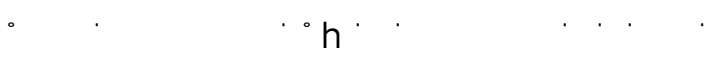

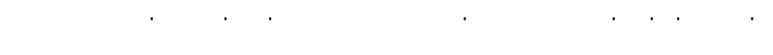

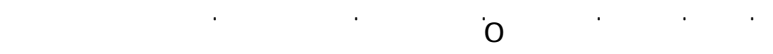

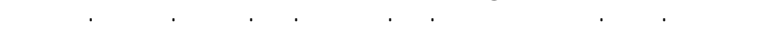

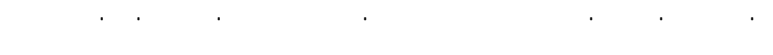

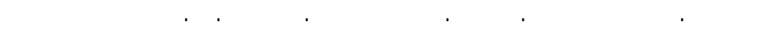

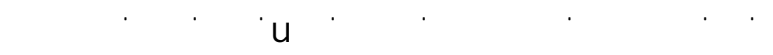

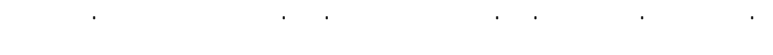

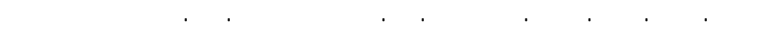

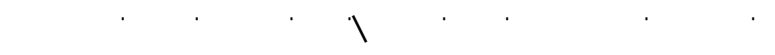

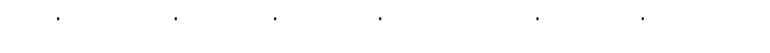

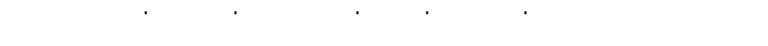

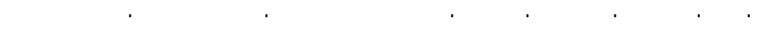

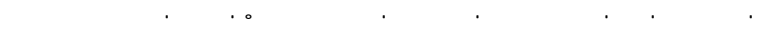

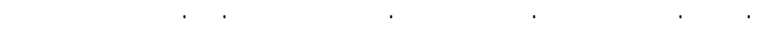

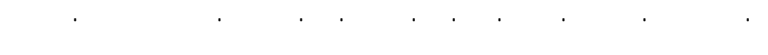

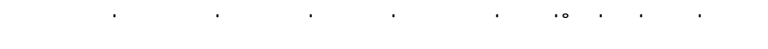

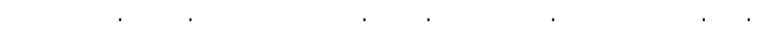

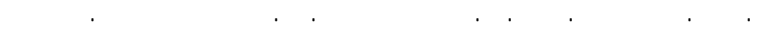

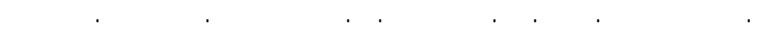

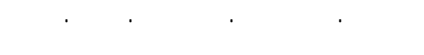




\section{CASE PRESENTATION}

A 20-year-old primipara was admitted at 32 weeks of gestation with severe pain confined to the upper abdomen and nausea, symptoms that started 2 days before. She had no history of previous illnesses. The patient was conscious, oriented and cooperative. On examination, she was afebrile, pulse rate was $98 / \mathrm{min}$, blood pressure $110 / 60$ and respiratory rate $23 / \mathrm{min}$. Physical examination revealed epigastric tenderness. Uterus was 32 weeks gravid size, relaxed. Fetal heart tones were at $145 / \mathrm{min}$. Laboratory tests done on the day of admission showed a white blood count of $15,200 / \mathrm{mm} 3$, and a platelet count of 282,000 . Liver function tests showed alkaline phosphatase - 135 U/L (ref: 55-170 U/I).Random blood sugar and renal function tests werewithin normal limits. Serum amylase was $172 \mathrm{U} / \mathrm{I}$ (ref: $<80 \mathrm{IU} / \mathrm{I})$, total cholesterol $208 \mathrm{mg} / \mathrm{dl}$ (ref:<200 mg/dl). Urine analysis was normal.

Abdominal ultrasonography showed a single live intrauterine fetus of 32 weeks gestational age with normalcardiac activity and adequate liquor. Abdominal ultrasonography revealed gallbladder with multiple gallstones with diameters about 7 $\mathrm{mm}$, extrahepatic biliary ducts about $14 \mathrm{~mm}$ with a hyperecogenic image about $7 \mathrm{~mm}$; no pathological findings have been noticed over pancreas at that time.

Imagistic follow-up has been performed via repeated ultrasound examination of the abdomen no complications have been found during the next days, 48 hours from admission the patient had normal values for extrahepatic biliary ducts diameters with no more stone images inside. Abdominal CT-scan was not used for diagnosis.

The patient was managed with intravenous fluids, analgesics and by nil per oral. We monitored the fetus using the non-stress test based on amniotic fluid volume. The test results for the fetus were well for gestational age. The mother improved with this supportive treatment within three days and started a soft diet without any symptoms. Serum amylase levels decreased to 87 IU/I on the 4th hospital day. The patient was discharged on the 6th hospital day in good condition.

The patient was followed for 10 day after discharge and was determined to be in good health. She delivered a viable infant by normal spontaneous vaginal delivery at $39 \mathrm{wk}$ of pregnancy. The newborn had good Apgar scores of 9 the baby's birth weight was $3.245 \mathrm{~kg}$. The newborn's physical examination and clinical findings were normal. The patient has maintained her health and had not experienced a recurrence of acute pancreatitis when assessed at 10 day after discharge and 6 weeks postpartum.

\section{DISCUSSION}

Acute pancreatitis in pregnancy remains a challenging clinical problem to manage, with a relatively limited but expanding evidence base. The relationship between acute pancreatitis and pregnancy remains unclear. Pregnancy does not primarily predispose pregnant woman to pancreatitis, but it does increase the risk of cholelithiasis and biliary sludge formation. AP in pregnancy is most often associated with gallstone disease or hypertriglyceridemia. Gallstones are the most common cause of acute pancreatitis during pregnancy responsible for more than $70 \%$ of cases (5). The incidence of gallstone related diseases including acute cholecystitis and biliary pancreatitis complicating pregnancy is $0.05-0.8 \%$ (6). Patients with known hypertriglyceridemia or family history should be followed up more closely because any delay can cause disastrous conclusions for mother and fetus (7). The hypertriglyceridemia can be attributed to increased estrogen due to pregnancy and the familial tendency for some women toward high triglyceride levels. Lipids and lipoprotein levels are increased during pregnancy, which increase three-fold peak in the third trimester (8). Identification of a biliary etiology for AP is important because as in the non-pregnant patient recurrence of AP episodes will occur in one-third to two-thirds of patients unless gallstones are removed (9). As well, in the third trimester, an enlarged uterus and increased intra-abdominal pressure on the biliary duct bring about acute pancreatitis more frequent (10). Recently, pancreatitis has been linked to more than 800 mutations of the cystic fibrosis transmembrane conductance regular gene (5).

The symptoms include epigastric pain (colicky or stabbing) which may radiate to the right flank, scapula and shoulder. Onset of pain is rapid, with maximal intensity in 10 to 20 minutes. Pain is steady and moderate to severe. Band-like radiation of the pain to the back occurs in half of patients. Other symptoms of gallbladder disease include anorexia, nausea, vomiting, dyspepsia, low-grade fever, tachycardia and fatty food intolerance (5).

The diagnosis of AP during pregnancy can be difficult. Pregnancy linked hematological and biochemical alterations may have an impact on the interpretation of the diagnostic tests. Diagnostic blood tests are essential for diagnosing acute pancreatitis and the tests can include serum amylase, 
lipase, complete blood count, serum triglycerides, calcium and liver function tests. An elevated serum amylase level has a diagnostic sensitivity of $81 \%$ and adding serum lipase increases this sensitivity to $94 \%$. Amylase levels do not correlate with disease severity. Elevated serum lipase levels stay elevated longer than amylase following an episode of pancreatitis (11).

Abdominal ultrasound is an excellent imaging technique for diagnosing acute pancreatitis in pregnancy because it has no associated radiation risk and is useful for detecting dilated pancreatic ducts and pseudocysts. Gallstones as a potential cause of AP are identified by abdominal ultrasound in most cases (12). However, it is insensitive for the detection of common bile duct stones or sludge. When a common bile duct stone is suspected based on abdominal ultrasound or biochemical abnormalities endoscopic ultrasound (EUS), a semi-invasive procedure of the biliary tree is an accurate modality for detecting common bile duct stones (13). Endoscopic ultrasound can be considered the best imaging study to evaluate common bile duct, although not for gallbladder stones. EUS requires expensive equipment, intravenous sedation and technical expertise. EUS is adequate prior to the consideration of therapeutic ERCP in patients where non-invasive imaging such as magnetic resonance cholangiopancreatography (MRCP) is not available, contraindicated or inconclusive.EUS have a high positive predictive value approaching $100 \%$ in detecting common bile duct stones and in many instances EUS is superior to MRCP (14).

Magnetic resonance imaging (MRI) and MRCP give multi-planar large field of view images of the body with excellent soft-tissue contrast and images of biliopancreatic duct systems. MRCP does not require any contrast injections and present no risk of renal injury. MRCP is a preferred method of evaluating $C B D$ in many clinical situations. There is paucity of data on the safety of MRI in the first trimester of pregnancy (15). Some authors have raised concerns of thermal injury to the fetus in first trimester (16). According to the Safety Committee of the Society for Magnetic Resonance Imaging, MR procedures are indicated in pregnant women if other non-ionizing forms of diagnostic imaging studies are inappropriate, or if the examination provides important information that would otherwise require exposure to ionizing radiation (17).

CT scan of the abdomen is the most commonly utilized imaging modality in diagnosing and later on in assessing severity of AP among adults. It is not recommended in pregnant patients because of the fear of radiation exposure to the fetus. In general $\mathrm{CT}$ is not the favourite modality of imaging in all trimesters of pregnancy in view of a small radiation risk to the fetus (18).

Ranson and Balthazar criteria for the classification of AP's severity in non-pregnant patients are often utilized as a guide to evaluate the disease's severity and treat it during pregnancy (19).

The fetal risks from AP during pregnancy are threatened preterm labor, prematurity and in utero fetal death. Previously reported high perinatal mortality rates secondary to AP are due to neonatal deaths after preterm delivery. In recent series, perinatal mortality rates were improved, mainly because $74 \%$ of the infants were delivered in term. Cases of acute biliary pancreatitis were associated with better perinatal outcomes than non-biliary causes (20).

Conservative treatment can be applied for mild pancreatitis, nevertheless, nutrition from long period of low fat diet consumption during treatment may not meet the requirements for normal fetal development. This therapy which includes gastric decompression, antispasmodic drugs, and antibiotics, is the first choice therapy, and the efficacy of surgical treatment remains controversial.

Regarding therapeutic strategy, no standardized guidelines have been published concerning the most effective management of acute biliary pancreatitis in pregnant women to reduce maternal and neonatal mortality and morbidity. Ducarme et al. (21) propose the following:

- First trimester: conservative treatment and laparoscopic cholecystectomy during second trimester

- Second trimester: laparoscopic cholecystectomy

- Third trimester: conservative treatment or ERCP with biliary endoscopic sphincteroto$\mathrm{my}$, and laparoscopic cholecystectomy in the early postpartum period

No formal recommendations have been published concerning the most effective management of hypertriglyceridemia-induced AP during pregnancy. Therapy should include a multidisciplinary team to address dietary fat restriction, appropriate nutritional supplements and possible medications when needed. Therapeutic plasma exchange and/or other treatment options including combined parenteral heparin and insulin infusions in severe cases are effective approaches to treat gestational hypertriglyceridemia-induced AP. Termination of pregnancy would be discussed according to the term and the severity of the AP. The early 
application of treatments in parturient women with hypertriglyceridemia-induced AP may improve the clinical course (22).

No definite guidelines have been published concerning the most effective management of chronic alcoholic pancreatitis during pregnancy. Therapeutic strategy should include a multidisciplinary team to address appropriate nutritional supplements, and possible medications when needed. Termination of pregnancy would be discussed according to the term and the severity of the recurrence of AP. The early application of treatments in parturient women with chronic alcoholic pancreatitis may improve the clinical course (23).

Acute fatty liver of pregnancy is a rare condition occurring in the third trimester of pregnancy, characterized by nausea, vomiting, abnormal and moderate hyperbilirubinemia. It may be complicated by renal and acute liver failure, respiratory distress, coagulopathy and rarely pancreatitis. Twelve cases of acute fatty liver of pregnancy associated with AP have been reported with a high maternal mortality rate (17\%) (24).

The topic of prophylactic use of antibiotics is very controversial and the choice of antibiotic in pregnancy is difficult.A systematic review and meta-analysis show antibiotic prophylaxis does not reduce mortality or protect against infected necrosis and frequency of surgical intervention (25).

The timing of pregnancy termination for patients with SAP has long been an issue for obstetricians.

No standardized guidelines have been published concerning the most effective way for delivery in women with AP during the third trimester of pregnancy to reduce maternal and neonatal mortality and morbidity. The decision depends on the gestational age and the severity of AP. When vaginal delivery is possible, it is preferable to limit the risk of superinfection necrosis associated with laparotomy used for cesarean sections. A multidisciplinary approach involving gastroenterologists, surgeons and obstetricians is desirable (26).
Indications for pregnancy termination can include full-term gestation, deteriorated condition after 24-48 hours of treatment, no improvement of paralytic ileus, stillbirth, fetal malformation, and severe pancreatitis. Higher levels of inflammatory cytokines in AP patients stimulate the uterus and cause abnormal uterine contractions, which could result in premature labor and preterm birth. Pregnancy should be terminated as soon as possible once acute hemorrhagic pancreatitis or hyperlipidemic pancreatitis is identified during pregnancy because these complications will raise the maternal and fetus mortality significantly. Cesarean section still is the preferred method for pregnancy termination in this case (27).

\section{CONCLUSIONS}

Acute pancreatitis during pregnancy is a rare but severe disease and remains a challenging clinical problem to manage, with a relatively limited base. There is no consensus in the literature regarding the management of AP during pregnancy. The general management of AP in pregnancy is supportive. From the various etiological factors for $A P$ in pregnancy, gallstone disease is the most common one.

Appropriate timing for termination of pregnancy is necessary and safe for acute pancreatitis in pregnancy patients. More studies are necessary for further elucidation of the etiology, risk factors and treatments of acute pancreatitis in pregnancy, mainly the severe type.

\section{Acknowledgement}

All authors contributed equally to the manuscript, meeting all 4 criteria of ICMJE recommendations: conception of the article, acquisition, analysis and interpretation of the data; drafting and revising the work; final approval of the version to be published; agreement to be accountable for all aspects of the work.

Conflict of interest: none declared Financial support: none declared

\section{REFERENCES}

1. Pitchumoni CS, Yegneswaran B. Acute pancreatitis in pregnancy. World $\mathrm{J}$ Gastroenterol. 2009;15(45):5641-5646.

2. Pandey M, Shukla VK. Lifestyle, parity, menstrual and reproductive factors and risk of gallbladder cancer. Eur J Cancer Prev. 2003;12:269-272
3. Igbinosa O, Poddar S, Pitchumoni C (2013) Pregnancy associated pancreatitis revisited. Clin Res Hepatol Gastroenterol 37: 177-181.

4. Eddy JJ, Gideonsen MD, Song JY, Grobman WA, O'Halloran P. Pancreatitis in pregnancy. Obstet Gynecol 2008; 112: 1075-1081.
5. Ramin KD, Ramin SM, Richey SD, Cunningham FG. Acute pancreatitis in pregnancy. Am J Obstet Gynecol. 1995; 173:187-191.

6. Ko CW. Risk factors for gallstone-related hospitalization during pregnancy and the postpartum. Am J Gastroenterol. 2006; 101:2263-2268. 
7. Yalcin Bahat P. Abruptio Placentae Caused by Hypertriglyceridemia-Induced AcutePancreatitis during Pregnancy: Case Report and Literature Review. Case Rep Obstet Gynecol. 2018 Sep 5;2018:3869695.

8. Knopp RH, Warth MR, Carrol CJ. Lipid metabolism in pregnancy: Changes in lipoprotein, triglyceride and cholesterol in normal pregnancy and the effects of diabetes mellitus. J Reprod Med. 1973; 10:95101.

9. Hernandez A, Petrov MS, Brooks DC, Banks PA, Ashley SW, Tavakkolizadeh A. Acute pancreatitis and pregnancy: A 10-year single center experience. J Gastrointest Surg. 2007;11:1623-1627.

10. Tang SJ, Rodriguez-Frias E, Singh S, Mayo MJ, Jazrawi SF, Sreenarasimhaiah J, et al. Acute pancreatitis during pregnancy. Clin Gastroenterol Hepatol. 2010; 8:85-90.

11. Sahu S, Raghuvanshi S, Bahl DV, Sachan P. Acute Pancreatitis In Pregnancy. Int J Surg. 2006; 11:Number2.

12. Block P, Kelly TR. Management of gallstone pancreatitis during pregnancy and the postpartum period. Surg Gynecol Obstet. 1989;168:426-428.

13. Yusuf TE, Bhutani MS. Role of endoscopic ultrasonography in diseases of the extrahepatic biliary system. J Gastroenterol Hepatol. 2004;19:243-250.
14. Lee YT, Chan FK, Leung WK, Chan HL, Wu JC, Yung MY et al. Comparison of EUS and ERCP in the investigation with suspected biliary obstruction caused by choledocholithiasis: A randomized study. Gastrointest Endosc. 2008;67:660-668.

15. Baker PN, Johnson IR, Harvey PR, Gowland PA, Mansfield P. A three-year follow-up of children imaged in utero with echo-planar magnetic resonance. $A m \mathrm{~J}$ Obstet Gynecol. 1994;170:32-33.

16. Levine D, Zuo C, Faro CB, Chen Q. Potential heating effect in the gravid uterus during MR HASTE imaging. J Magn Reson Imaging. 2001;13:856-861.

17. Shellock FG, Kanal E. Policies, guidelines, and recommendations for MR imaging safety and patient management. SMRI Safety Committee. J Magn Reson Imaging. 1991;1:97-101.

18. Kennedy A. Assessment of acute abdominal pain in the pregnant patient. Semin Ultrasound CT MR. 2000;21:64-77.

19. Ranson JH. Etiological and prognostic factors in human acute pancreatitis: A review. Am J Gastroenterol 1982; 7: 633-638.

20. Eddy JJ, Gideonsen MD, Song JY, Grobman WA, O'Halloran P. Pancreatitis in pregnancy. Obstet Gynecol 2008; 112: 1075-1081.
21. G Ducarme, F Maire, P Chatel, D Luton and $P$ Hammel. Acute pancreatitis during pregnancy: a review. Journal of Perinatology 2014, 34:87-94\& 2014 Nature America.

22. Goldberg AS, Hegele RA. Severe hypertriglyceridemia in pregnancy. $\mathrm{J}$ Clin Endocrinol Metab 2012; 97: 2589-2596.

23. Stimac D, Stimac T. Acute pancreatitis during pregnancy. Eur J Gastroenterol Hepatol 2011; 23: 839-844.

24. Moldenhauer JS, O'Brien JM, Barton JR, Sibai B. Acute fatty liver of pregnancy associated with pancreatitis: A lifethreatening complication. Am J Obstet Gynecol 2004; 190: 502-505.

25. Jafri N. S., Mahid S. S., Idstein S. R., Hornung C. A., Galandiuk S. Antibiotic prophylaxis is not protective in severe acute pancreatitis: A systematic review and meta-analysis. The American Journal of Surgery. 2009;197(6):806-813.

26. Ducarme G, Chatel P, Alves A, Hammel P, Luton $D$. Management of necrotizing pancreatitis in the third trimester of pregnancy. Arch Gynecol Obstet 2009; 279: 561-563.

27. Yanmei Sun. Clinical analysis of 16 patients with acute pancreatitis in the third trimester of pregnancy. Int J Clin Exp Pathol. 2013; 6(8): 1696-1701. 